\title{
Short Communication Efficacy of gene therapy-delivered cytosine deaminase is determined by enzymatic activity but not expression
}

\author{
L Dubois', T Dresselaers², W Landuyt ${ }^{3}$, K Paesmans', A Mengesha', BG Wouters', P Van Hecke², J Theys ${ }^{*}, 1,4$ \\ and $P$ Lambin ${ }^{1,3,4}$ \\ 'Department of Radiation Oncology (Maastro Lab), GROW Research Institute, University of Maastricht, UNS 50/23, PO Box 6I 6, 6200 MD, Maastricht, \\ The Netherlands; ' Biomedical NMR Unit, K.U. Leuven, Herestraat 49, PO Box 803, 3000 Leuven, Belgium; ' ${ }^{2}$ Laboratory of Experimental Oncologyl \\ Radiobiology, K.U. Leuven, Gasthuisberg-CDG 8th floor, Herestraat 49, 3000 Leuven, Belgium
}

\begin{abstract}
The potential utility of tumour-selective 5-fluorouracil treatment using attenuated Salmonella serovar typhimurium recombinant for cytosine deaminase (TAPET-CD) has been documented in experimental settings. The present data demonstrate that in vivo ${ }^{19} \mathrm{~F}$ magnetic resonance spectroscopy measurements allow the outcome prediction of this prokaryotic-based therapy, demonstrating the necessity of non-invasive real-time imaging techniques for treatment monitoring.

British Journal of Cancer (2007) 96, 758-76I. doi: I0.1038/sj.bjc.6603624 www.bjcancer.com

Published online 20 February 2007

(C) 2007 Cancer Research UK
\end{abstract}

Keywords: ${ }^{19}$ F-MRS; Salmonella typhimurium; gene therapy; cytosine deaminase; xenograft human tumour

Positron emission tomography and (nuclear) magnetic resonance imaging (MRI) have been introduced to evaluate the biological characteristics of tumours and to monitor the efficacy of treatment on an individual basis. Fluor-containing chemotherapeutics, such as 5-fluorouracil (5-FU) and its metabolites, can be detected noninvasively in tumour and liver tissues using ${ }^{19} \mathrm{~F}$-magnetic resonance spectroscopy ( $\left.{ }^{19} \mathrm{~F}-\mathrm{MRS}\right)$. Preclinical animal investigations and patient studies suggest that trapping of 5-FU in the tumour correlates with response and may provide prognostic information on treatment outcome (Wolf et al, 1990; Schlemmer et al, 1999; Martino et al, 2005).

Ample data exist on the use of the cytosine deaminase (CD) enzyme, which converts the nontoxic 5-fluorocytosine (5-FC) into the cytostatic 5-FU, in a number of experimental settings. Among these, we and others have demonstrated that recombinant bacteria such as Clostridium and attenuated Salmonella, can be used to transfer CD selectively to the rodent tumour microenvironment, resulting in antitumour effects (Minton et al, 1995; Theys et al, 2001; King et al, 2002; Mei et al, 2002). In addition, we reported the feasibility of ${ }^{19} \mathrm{~F}$-MRS to assess non-invasively the intratumour conversion of 5-FC into 5-FU mediated by CD-recombinant Salmonella (Dresselaers et al, 2003).

The aim of the present study was to investigate the value of in vivo ${ }^{19} \mathrm{~F}$-MRS to predict the success of the TAPET-CD/5-FC cancer therapy system and to evaluate it as a tool to allow individualised treatment.

\section{MATERIALS AND METHODS}

HCT116 human colorectal carcinoma cells were xenografted $\left(1.5 \times 10^{6}\right.$ cells per $\left.100 \mu \mathrm{l}\right)$ subcutaneously at the lower flank of

*Correspondence: Dr J Theys; E-mail: jan.theys@maastro.unimaas.nl

${ }^{4}$ These two authors contributed equally to this work

Revised 10 January 2007; accepted 12 January 2007; published online 20

February 2007
8- to 10 -week-old female NMRI nu/nu mice. Treatment and ${ }^{19} \mathrm{~F}$ MRS measurements started when tumour volumes reached $>300 \mathrm{~mm}^{3}$. Tumours were measured in three orthogonal dimensions with a calliper and volumes were calculated according to the formula $A B C \times \pi / 6$

TAPET-CD (VION Pharmaceuticals Inc., New Haven, CT, USA), in detail described by Zheng et al (2000), was cultured as described previously (Mei et al, 2002) and $2.0 \times 10^{6}$ bacteria were injected intravenously (tail vein). Six days after TAPET-CD administration, 5 -FC was daily administered intraperitoneally in one group of animals $(n=12)$, whereas a control group $(n=4)$ received saline according to the same schedule.

To determine the intratumour conversion activity of the TAPET-CD, in vivo ${ }^{19} \mathrm{~F}$-MRS experiments were performed in a 4.7 T BIOSPEC horizontal magnet (Bruker), equipped with a double-tuned $\left({ }^{19} \mathrm{~F}-{ }^{1} \mathrm{H}\right)$ surface coil of $1 \mathrm{~cm}$ diameter, as described previously (Dresselaers et al, 2003). ${ }^{19} \mathrm{~F}$-MRS measurements were performed four times on each animal, starting 6 days after TAPET$\mathrm{CD}$ injection and then every sixth or seventh day. An additional 5 -FC injection was given immediately before the ${ }^{19} \mathrm{~F}-\mathrm{MRS}$ measurements.

At the end of the follow-up period, animals were killed, tumours excised and homogenates were made to quantify bacterial colonisation, presence (Western blotting) and activity (thin-layer chromatography (TLC)) of CD, according to Theys et al (2001). Briefly, Western blotting was carried out by loading $25 \mu \mathrm{g}$ of tumour cell extracts on the gel. Anti-CD monoclonal antibody (MTM Laboratories Inc., Westborough, MA, USA) in a 1:5000 dilution was used as primary antibody. After overnight incubation of the sonicated lysates with 5-FC $\left(10 \mathrm{mg} \mathrm{ml}^{-1}\right)$, the conversion into 5-FU was analysed by TLC on silica gel plates eluted with butanol. 5-Fluorocytosine and 5-FU were loaded as reference. Histological evaluation of hypoxia and necrosis was performed on paraffin-embedded tumour pieces after administration of the exogenous hypoxia marker pimonidazole. Staining and evaluation 
A

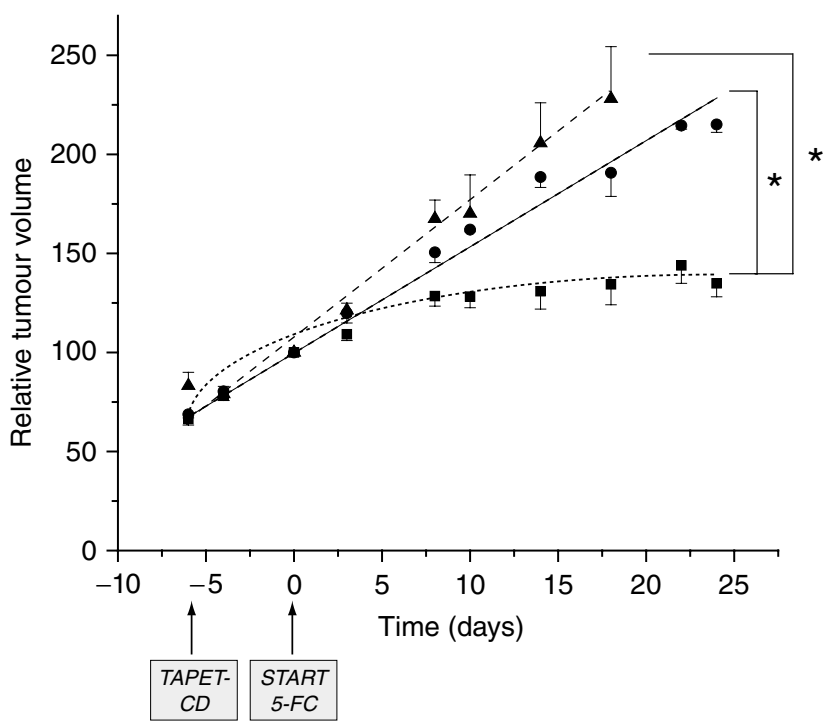

C

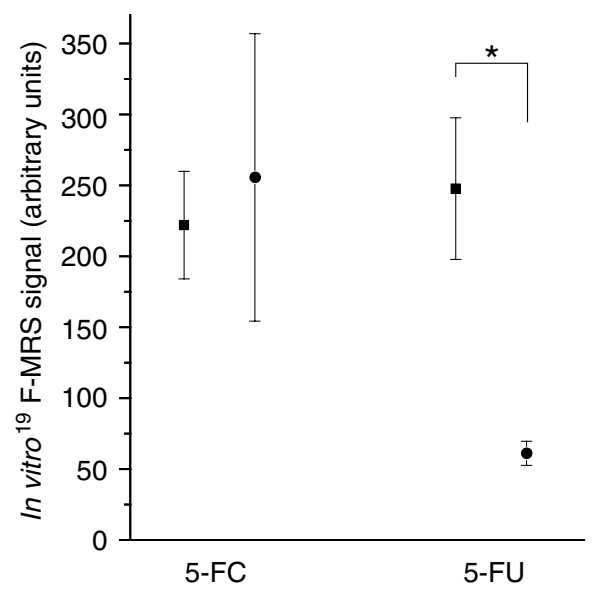

B
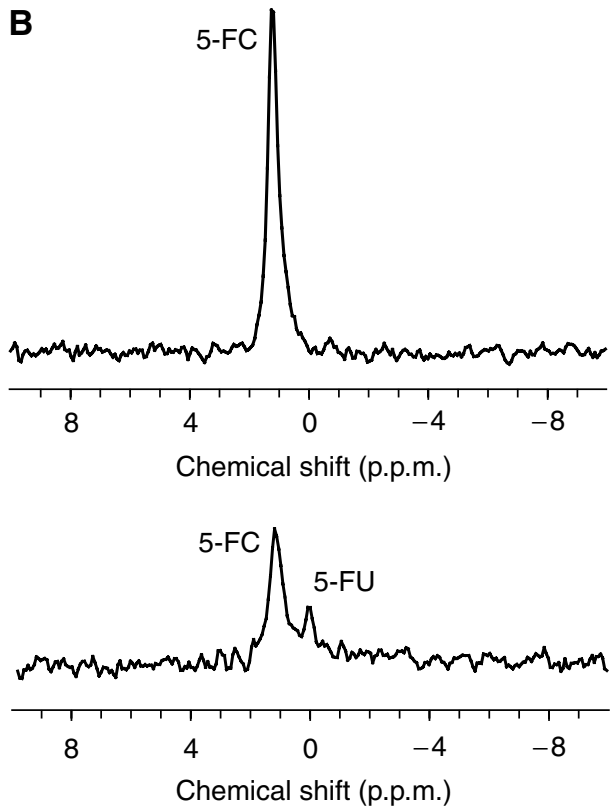

D

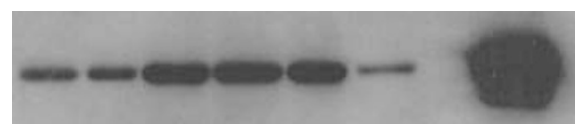

$\begin{array}{lllllllll}\text { NR1 } & \text { R6 } & \text { NR2 } & \text { R7 } & \text { R8 } & \text { NR3 } & -\mathrm{C} & \text { +C }\end{array}$

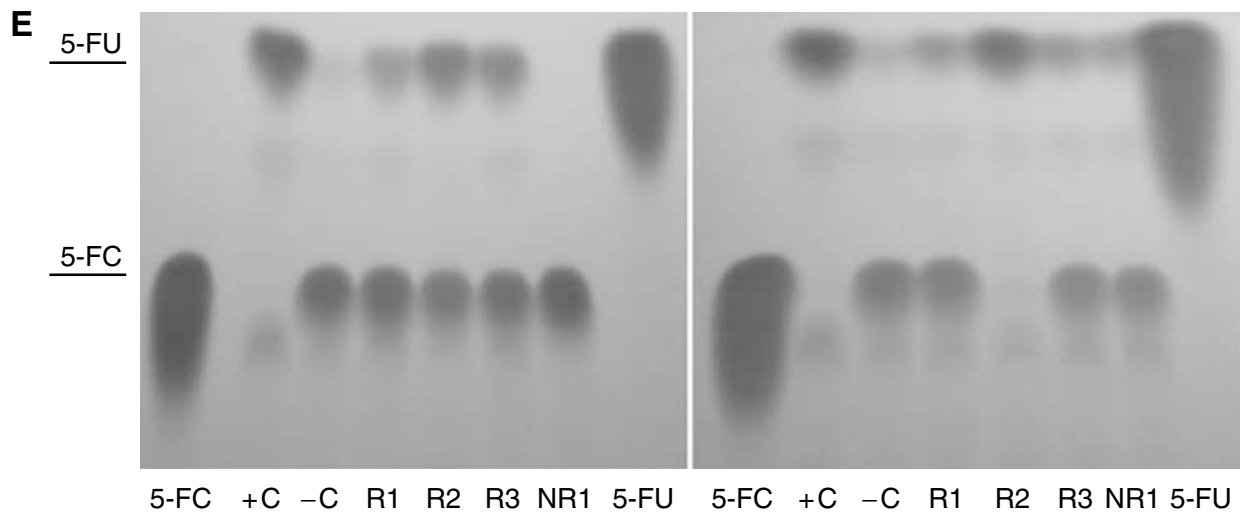

Figure I (A) Growth delay for 'responder' $(9 / 12 ; \mathbf{\square})$ and 'nonresponder' $(3 / 12 ; \boldsymbol{O})$ animals. Sham saline-treated animals are indicated with $\boldsymbol{\Delta}$. Animals in which a 5-FU signal could be detected using in vivo ' $\mathrm{F}$-MRS during the follow-up period were marked as 'responders', the others as 'nonresponders'. The $100 \%$ value represents the tumour volume at day 0 (ie volume at the start of 5-FC administration). Statistical significance at $P<0.00$ I using linear regression analysis is indicated (*). Data are shown as mean \pm s.e.m. (B) Representative spectra ( 3 min/spectrum: TR=0.75 s; NA $=1024$; LB =6 Hz; zerofilling to 4096 points) of a 'nonresponder' (top) and a 'responder' (bottom) animal. (C) Quantification of the amount of 5-FC and 5-FU using in vitro ${ }^{19} \mathrm{~F}-\mathrm{MRS}$ on perchloric acid tumour extracts of 'responders' (- $)$ and 'nonresponders' $(\mathbf{O})$. Statistical significance at $P<0.05$ using a nonparametric Mann-Whitney $U$ test is indicated (*). Data are shown as mean \pm s.e.m. (D-E) Western blotting and thin-layer chromatography (TLC) for CD expression and activity respectively in tumour homogenates from HCTII6 xenograft In NMRI nu/nu mice injected with TAPET-CD. TLC was done before (E left) and after (E right) adding Luria-bertani medium to the tumour extracts with overnight incubation. 'Responders' are indicated with R, 'nonresponders' with NR. The $+C$ indicates a sample of bacterial lysate from in vitro growing TAPET-CD and represent the positive control. $-\mathrm{C}$ represents the negative control, that is, bacterial lysate from the in vitro growing PC0698 strain, which does not encode CD. 
of the sections were performed as described previously (Dubois et al, 2004).

In vitro ${ }^{19} \mathrm{~F}-\mathrm{MRS}$ analysis was performed on perchloric acid extracts using a AMX360 (8.4 T) spectrometer (Bruker, Karlsruhe, Germany; Dresselaers et al, 2003).

All animal experiments were in agreement with national guidelines, approved by the Animal Ethics Committee of the University 'KU Leuven', Belgium, and procedures were according to the guidelines defined by the UKCCCR (Workman et al, 1998).

\section{RESULTS}

${ }^{19}$ F-MRS measurements were performed repeatedly on every animal starting at 6 days after TAPET-CD administration and immediately after the 5-FC injection. These spectroscopy analyses allowed us to evaluate the in vivo intratumour conversion of 5-FC into 5-FU, which resulted from TAPET-CD activity. Animals in which a 5-FU signal could be detected during the follow-up period were marked as 'responders' (9 out of 12), the others as 'nonresponders' (3 out of 12). Linear regression analysis showed a significant tumour growth delay for the 'responders' compared to the 'non-responders' (29.00 days; $\beta=5.72 ; P<0.001)$ and control saline-treated group ( 29.83 days; $\beta=3.32 ; P<0.001$ ), as witnessed from the interaction between time and group (Figure 1A). Representative spectra of 'responder' and 'non-responder' animals are shown in Figure 1B. The final in vivo measurements were subsequently confirmed by quantification of the 5-FU amount using in vitro ${ }^{19} \mathrm{~F}$-MRS analyses. Whereas equal amounts of 5-FC were found, significant differences in 5-FU amount existed between 'responders' and 'non-responders' $(P=0.044)$ (Figure 1C). This was not caused by a different level of colonisation because the number of viable bacteria present in the tumours was not significantly different in both groups (equally high colonisation, $>10^{9} \mathrm{cfu} \mathrm{g}^{-1}$ tumour tissue). In addition, Western blotting on tumour extracts did not reveal differences in $C D$ protein expression (Figure 1D). To exclude presence of mutations within the CD protein, lysates were made from bacteria isolated out of tumours from both 'responders' and 'non-responders'. TLC experiments with these bacterial lysates demonstrated conversion of 5-FC to 5-FU in all cases, indicating a fully functional $\mathrm{CD}$ protein (data not shown). However, the ability for conversion of 5FC into 5-FU, as determined by TLC experiments on tumour extracts, could only be shown in the 'responders' and not in the 'non-responders' group (Figure 1E left). This corresponds to the very small amount of 5-FU detected in the 'non-responder animals' using the more sensitive in vitro ${ }^{19} \mathrm{~F}$-MRS (Figure $1 \mathrm{C}$ ). Interestingly, the 'no-conversion phenomenon' in the 'non-responder' animals could be reversed by adding nutrients (Luria-Bertani medium) to the tumour extracts and allowing the mixture incubate overnight before making sonicated lysates (Figure 1E, right).

\section{DISCUSSION}

The present study shows that the dynamic 5-FC/5-FU conversion in the human colorectal HCT116 tumour xenograft following systemic injection of TAPET-CD and 5-FC can be monitored using in vivo ${ }^{19} \mathrm{~F}$-MRS. More importantly, we demonstrate the need for sequential non-invasive ${ }^{19} \mathrm{~F}-\mathrm{MRS}$ imaging as a tool for TAPET-CD/ 5-FC therapy prediction. Given the fact that a pilot clinical trial with TAPET-CD, in which two out of three refractory cancer patients maintained stable disease when functional $\mathrm{CD}$ was produced within the tumour (Nemunaitis et al, 2003) have already started, our results point to the importance of not only monitoring tumour colonisation but also CD functionality.

Tumours in which a 5-FU signal could be detected using in vivo ${ }^{19} \mathrm{~F}-\mathrm{MRS}$ during the follow-up period were marked as 'responders', the others were marked 'non-responders'. Despite similar TAPET$\mathrm{CD}$ colonisation levels in all tumours, growth delay assays showed a significant difference in success of the TAPET-CD/5-FC therapy for the 'responders' vs 'non-responders' and the control salinetreated animals. In vitro ${ }^{19} \mathrm{~F}$-MRS analysis performed on the perchloric acid extracts of the resected tumours taken at the end of the follow-up period, confirmed the in vivo ${ }^{19} \mathrm{~F}-\mathrm{MRS} 5-\mathrm{FC} / 5-\mathrm{FU}$ measurements. Intriguingly, Western blotting did not reveal a difference in $\mathrm{CD}$ protein expression between the samples of 'responders' and 'non-responders,' and TLC analysis on bacterial lysates confirmed the CD functionality in all cases. This indicates that in the TAPET-CD present in the tumour, the CD gene was not selectively lost. In other words, expression of CD by recombinant bacteria in the tumour is not enough to establish an antitumour effect when combined with 5-FC administration. Kamm et al (1996) have argued that differences in 5-FU levels could be explained by morphological heterogeneity in tumours. However, we did not find histological differences in the amount of hypoxia/ necrosis between the tumours of 'responders' and 'non-responders' nor in the levels of 5-FC detected in the tumours via ${ }^{19}$ F-MRS (both in vitro and in vivo). These results showed that prodrug diffusion to the bacterial colonisation sites in both groups is not impaired. As no differences were seen in bacterial colonisation and $\mathrm{CD}$ protein expression, but significance was obtained in growth delay between 'responders' and 'non-responders', we reasoned that the observed difference was probably owing to either the presence of an inhibitory factor in the tumours of the 'non-responders', or to the lack of factors needed for CD enzyme activity. The catalytic activity of $\mathrm{CD}$ is shown to be dependent on $\mathrm{Fe}^{2+}$ or other divalent metal ions that function as cofactor. Although it might be possible that these cofactors are present in varying amounts among tumours, this appeared not to be limiting in our study (data not shown). Alternatively, low intratumoural $\mathrm{pH}$ or high endogenous cytosine concentrations that compete with 5-FC for the active site might reduce CD activity. However, as TLC experiments revealed that adding fresh growth medium to the tumour extracts of nonresponders resulted in 5-FC/5-FU conversion, our data point towards the absence of yet other necessary factors. From our results, it is apparent that in a subgroup of tumours, the absence of these necessary nutrients for efficient $C D$ enzymatic activity can result in a lack of antitumour efficacy. Therefore, our results stress the importance of non-invasive real-time imaging tools as ${ }^{19} \mathrm{~F}$-MRS for individualised treatment as it allows the prediction of antitumour efficacy when using TAPET-CD/5-FC therapy or related treatments.

\section{ACKNOWLEDGEMENTS}

This work was financially supported by the LSCH-CT-2003502932/Euroxy 6th framework grant (LD) and the Vanderes Foundation (AM). We thank Ruud Houben for statistical assistance.

\section{REFERENCES}

Dresselaers T, Theys J, Nuyts S, Wouters B, de Bruijn E, Anne J, Lambin P, Van Hecke P, Landuyt W (2003) Non-invasive ${ }^{19} \mathrm{~F}$ MR spectroscopy of 5-fluorocytosine to 5-fluorouracil conversion by recombinant Salmonella in tumours. Br J Cancer 89: 1796-1801 
Dubois L, Landuyt W, Haustermans K, Dupont P, Bormans G, Vermaelen P, Flamen P, Verbeken E, Mortelmans L (2004) Evaluation of hypoxia in an experimental rat tumour model by [(18)F]fluoromisonidazole PET and immunohistochemistry. Br J Cancer 91: 1947-1954

Kamm YJ, Heerschap A, Rosenbusch G, Rietjens IM, Vervoort J, Wagener DJ (1996) 5-Fluorouracil metabolite patterns in viable and necrotic tumor areas of murine colon carcinoma determined by $19 \mathrm{~F}$ NMR spectroscopy. Magn Reson Med 36: 445-450

King I, Bermudes D, Lin S, Belcourt M, Pike J, Troy K, Le T, Ittensohn M, Mao J, Lang W, Runyan JD, Luo X, Li Z, Zheng LM (2002) Tumortargeted Salmonella expressing cytosine deaminase as an anticancer agent. Hum Gene Ther 13: 1225-1233

Martino R, Gilard V, Desmoulin F, Malet-Martino M (2005) Fluorine-19 or phosphorus-31 NMR spectroscopy: a suitable analytical technique for quantitative in vitro metabolic studies of fluorinated or phosphorylated drugs. J Pharm Biomed Anal 38: 871-891

Mei S, Theys J, Landuyt W, Anne J, Lambin P (2002) Optimization of tumor-targeted gene delivery by engineered attenuated Salmonella typhimurium. Anticancer Res 22: $3261-3266$

Minton NP, Mauchline ML, Lemmon MJ, Brehm JK, Fox M, Michael NP, Giaccia A, Brown JM (1995) Chemotherapeutic tumour targeting using Clostridial spores. FEMS Microbiol Rev 17: 357-364

Nemunaitis J, Cunningham C, Senzer N, Kuhn J, Cramm J, Litz C, Cavagnolo R, Cahill A, Clairmont C, Sznol M. (2003) Pilot trial of genetically modified, attenuated Salmonella expressing the E. coli cytosine deaminase gene in refractory cancer patients. Cancer Gene Ther 10: 737-744

Schlemmer HP, Becker M, Bachert P, Dietz A, Rudat V, Vanselow B, Wollensack P, Zuna I, Knopp MV, Weidauer H, Wannenmacher M, van Kaick G (1999) Alterations of intratumoral pharmacokinetics of 5fluorouracil in head and neck carcinoma during simultaneous radiochemotherapy. Cancer Res 59: 2363-2369

Theys J, Landuyt W, Nuyts S, Van Mellaert L, van Oosterom A, Lambin P, Anne J (2001) Specific targeting of cytosine deaminase to solid tumors by engineered Clostridium acetobutylicum. Cancer Gene Ther 8: 294-297

Wolf W, Presant CA, Servis KL, el-Tahtawy A, Albright MJ, Barker PB, Ring III R, Atkinson D, Ong R, King M, Singh M, Ray M, Wiseman C, Blayney D, Shani J (1990) Tumor trapping of 5-fluorouracil: in vivo 19F NMR spectroscopic pharmacokinetics in tumor-bearing humans and rabbits. Proc Natl Acad Sci USA 87: $492-496$

Workman P, Twentyman P, Balkwill F, Balmain A, Chaplin D, Double J, Embleton J, Newell D, Raymond R, Stables J, Stephens T, Wallace J (1998) United Kingdom Co-ordinating Committee on Cancer Research (UKCCR) guidelines for the Welfare of Animals in Experimental Neoplasia (second edition). Br J Cancer 77: 1 - 10

Zheng LM, Luo X, Feng M, Li Z, Le T, Ittensohn M, Trailsmith M, Bermudes D, Lin SL, King IC (2000) Tumor amplified protein expression therapy: Salmonella as a tumor-selective protein delivery vector. Oncol. Res. 12: $127-135$ 